\title{
A NOTE ON INTERPOLATION
}

\author{
S. J. SIDNEY AND E. L. STOUT ${ }^{1}$
}

I. Let us say that $A$ is a uniform algebra on $X$ provided that $A$ is a point separating, uniformly closed subalgebra of $\mathfrak{e}(X)$ which contains the constants. The space $X$ is assumed to be a compact Hausdorff space. In this note we establish the following result.

Theorem. Let $A$ be a uniform algebra on $X$ and $E$ a closed subset of $X$. If $\operatorname{Re} A \mid E=\mathfrak{C}_{R}(E)$, then $A \mid E=\mathcal{C}(E)$.

This result should be compared with two theorems which appear in the literature. One of these, due to Wermer [W], states that if $A$ is a uniform algebra on $X$ such that $\operatorname{Re} A$ is an algebra, then $A=\mathrm{e}(X)$. The other, due to Hoffman and Wermer [HW] (see also [B]) says that if $A$ is a uniform algebra on $X$, then $A=\mathfrak{C}(X)$ if $\operatorname{Re} A$ is closed in $\mathfrak{C}_{R}(X)$. Either of these results applied to $(A \mid E)^{-}$implies that if $\operatorname{Re} A \mid E=\mathfrak{C}_{R}(E)$, then $A \mid E$ is dense in $\mathfrak{e}(E)$. However, our result does not seem to be a direct consequence of these facts.

The theorem has as a corollary a result which generalizes the Hoffman-Wermer theorem.

Corollary. Let $A$ be a uniform algebra on $X$. If $E \subset X$ is a closed set such that $\operatorname{Re} A \mid E$ is closed in $\mathfrak{C}_{R}(E)$, then $A \mid E=\mathfrak{C}(E)$.

Proof of the corollary. We have that $(\operatorname{Re} A) \mid E=\operatorname{Re}\left[(A \mid E)^{-}\right]$. Thus, the result of Hoffman and Wermer implies that $(A \mid E)^{-}=\mathrm{e}(E)$. Consequently $\operatorname{Re} A \mid E$ is dense in $\mathfrak{C}_{R}(E)$, and since, by hypothesis, it is closed, we have that $\operatorname{Re} A \mid E=\mathfrak{C}_{R}(E)$. The theorem now implies that $A \mid E=\mathfrak{e}(E)$.

II. Proof of the Theorem. By a result of Glicksberg [G, Corollary 3.2], we have that $A \mid E=\mathfrak{e}(E)$ if and only if there is a constant $C$ such that $\left\|\mu_{E}\right\| \leqq C\left\|\mu_{E^{c}}\right\|$ whenever $\mu$ is a complex regular Borel measure on $X$ which annihilates $A$. Here $\mu_{E}$ denotes the restriction of $\mu$ to $E, E^{c}$ the complement of $E$ in $X$.

Suppose no such constant $C$ exists so that for $n=1,2,3, \cdots$ there exists a measure $\mu^{n}$ orthogonal to $A$ which satisfies

$$
\left\|\mu_{E}^{n}\right\|>n\left\|\mu_{E}^{n}\right\|
$$

Received by the editors January 17, 1967.

1 Research supported in part by NSF Grant GP-5493. 
From this assumption we will derive a contradiction. We may assume that $\left\|\mu^{n}\right\|=1$.

Let $\mu=\sum_{n=1}^{\infty} 2^{-n}\left|\mu^{n}\right|$. Then $L^{\infty}=L^{\infty}(\mu)$ is a commutative Banach algebra with identity under the essential supremum norm and the almost everywhere pointwise operations. Define $B$ to be the algebra of pointwise limits of a.e. [ $\mu$ ] convergent bounded sequences in $A$, and let $H^{\infty}$ denote the norm closure of $B$ in $L^{\infty}$. This is not the usual definition of $H^{\infty}$; it was devised so that $H^{\infty}$ is a closed subalgebra of $L^{\infty}$ containing $A$ and having the important property that each $\mu^{n}$ annihilates it.

Since the map $f \rightarrow \operatorname{Re} f \mid E$ from $A$ to $\mathfrak{C}_{R}(E)$ is real-linear, continuous, and onto, the open mapping theorem guarantees the existence of a constant $C_{1}$ such that if $g \in \mathcal{C}_{R}(E)$, there is $f \in A$ with $\operatorname{Re} f \mid E=g$ and $\|f\|_{X} \leqq C_{1}\|g\|_{E}$. From this, it follows that $\operatorname{Re} H^{\infty}\left|E=L_{R}^{\infty}\right| E$ and in fact that $\operatorname{Re} B\left|E=L_{R}^{\infty}\right| E$. Let $g \in L_{R}^{\infty} \mid E$. There exists a bounded sequence $\left\{g_{n}\right\} \subset \mathcal{C}_{R}(E)$ with $g_{n} \rightarrow g$ a.e. $[\mu]$ on $E$. Then there is a bounded sequence $\left\{f_{n}\right\} \subset A$ with $\operatorname{Re} f_{n} \mid E=g_{n}$. The sequence $\left\{f_{n}\right\}$ is then a bounded set in $L^{2}(\mu)$ and so, by passing to a subsequence if necessary, we can assume that $f_{n}$ converges weakly to $f_{0} \in L^{2}(\mu)$. The Banach-Saks theorem [RN, p. 80] now implies that $\left\|h_{k}-f_{0}\right\|_{2} \rightarrow 0$ where $h_{k}=\left(f_{n_{1}}+\cdots+f_{n_{k}}\right) / k$ for some subsequence $\left\{f_{n_{j}}\right\}$ of $\left\{f_{n}\right\}$. A subsequence of $\left\{h_{k}\right\}$ then converges to $f_{0}$ pointwise a.e. [ $\left.\mu\right]$. The sequence $\left\{h_{k}\right\}$ is a bounded sequence in $A$, and thus $f_{0} \in B$. Finally, since $\operatorname{Re} f_{j} \mid E=g_{j}$ a.e. $[\mu]$, we see that $h_{k} \mid E \rightarrow g$ a.e. $[\mu]$, so $\operatorname{Re} f_{0} \mid E$ $=g$ a.e. $[\mu]$. Thus, $\operatorname{Re} H^{\infty}\left|E=L_{R}^{\infty}\right| E$ as asserted.

Let $\Gamma$ denote the maximal ideal space of $L^{\infty}$. Then $\Gamma$ is an extremally disconnected compact Hausdorff space [H, pp. 169-171] and is, therefore, an $F$-space. (See $[\mathbf{G J}]$ for a discussion of $F$-spaces and extremally disconnected spaces.) The Gelfand transform ${ }^{\wedge}: L^{\infty} \rightarrow \mathfrak{e}(\Gamma)$ is an isometric isomorphism with range the whole of $\mathfrak{e}(\Gamma)$, and under ${ }^{\wedge}, H^{\infty}$ goes onto a closed subalgebra of $\mathfrak{e}(\Gamma)$. The set $E$ corresponds to an open and closed subset $\hat{E}$ of $\Gamma$ by means of $\hat{\chi}_{E}=\chi \hat{E}$. Since $\operatorname{Re} H^{\infty}\left|E=L_{R}^{\infty}\right| E$, we have that $\operatorname{Re} \hat{H}^{\infty} \mid \hat{E}=\mathfrak{C}_{R}(\hat{E})$. Applying either the result of Wermer or that of Hoffman and Wermer cited above, we may conclude that $\hat{H}^{\infty} \mid \hat{E}$ is dense in $\mathcal{e}(\hat{E})$. A result of Badé and Curtis [BC, Theorem 3.3] now implies that $\hat{H}^{\infty} \mid \hat{E}=\mathfrak{C}(\hat{E})$, and consequently, we have $H^{\infty}\left|E=L^{\infty}\right| E$.

Define a function $\phi_{n} \in L^{\infty} \mid E$ by requiring that $\left|\phi_{n}\right|=1$ a.e. $[\mu]$ on $E$ and that $\phi_{n}=d \mu^{n} / d\left|\mu^{n}\right|$ a.e. $\left[\left|\mu^{n}\right|\right]$ on $E$. Since each $\phi_{n}$ is of modulus one a.e. $[\mu]$, there is a constant $C_{2}$ independent of $n$ such that for each $n$, there is $f_{n} \in H^{\infty}$ with $f_{n} \mid E=\phi_{n}{ }^{-1},\left\|f_{n}\right\| \leqq C_{2}$. (The existence of the constant $C_{2}$ is guaranteed by the open mapping theorem since the 
map $f \rightarrow f \mid E$ from $H^{\infty}$ to $L^{\infty} \mid E$ is continuous, linear and onto.) Since $\mu^{n}$ is orthogonal to $H^{\infty}$, we have

$$
0=\int f_{n} d \mu^{n}=\int_{E} \phi_{n}^{-1} d \mu^{n}+\int_{E c} f_{n} d \mu^{n} .
$$

Thus,

$$
\left\|\mu_{E}^{n}\right\|=\int_{E} \phi_{n}^{-1} d \mu^{n} \leqq\left\|f_{n}\right\|_{\infty}\left\|\mu_{E}^{n}\right\|
$$

whence

$$
\left\|\mu_{E}^{n}\right\| \leqq C_{2}\left\|\mu_{E c}^{n}\right\| .
$$

For large values of $n,(2)$ is inconsistent with (1), so we have derived a contradiction and established the theorem.

AdDEd IN PRoOF. The generalization of Wermer's result [W] analogous to our corollary is false without some sort of additional hypothesis. For let $X$ denote the closed unit disc in the complex plane, $A$ the subalgebra of $C(X)$ consisting of functions analytic on the interior of $X, E$ the intersection of $X$ with the real axis. Then $A \mid E$ is symmetric, hence $\operatorname{Re} A \mid E$ is closed under multiplication, but $A \mid E \neq C(E)$.

\section{REFERENCES}

[BC] W. G. Badé and P. C. Curtis, Embedding theorems for commutative Banach algebras, Pacific J. Math. 18 (1966), 391-407.

[B] A. Browder, "On a theorem of Hoffman and Wermer," in Function algebras, Scott, Foresman and Co., Chicago, Ill. 1966; pp. 88-89.

[GJ] L. Gillman and M. Jerison, Rings of continuous functions, Van Nostrand, Princeton, N. J., 1960.

[G] I. Glicksberg, Measures orthogonal to algebras and sets of antisymmetry, Trans. Amer. Math. Soc. 105 (1962), 415-435.

[H] K. Hoffman, Banach spaces of analytic functions, Prentice-Hall, Englewood Cliffs, N. J., 1962.

[HW] K. Hoffman and J. Wermer, A characterization of $\mathfrak{e}(X)$, Pacific J. Math. 12 (1962), 941-944.

[RN] F. Riesz and B. Sz.-Nagy, Functional analysis, Ungar, New York, 1955.

[W] J. Wermer, The space of real parts of a function algebra, Pacific J. Math. 13 (1963), 1423-1426.

YaLE UNIVERsity 